\title{
Risk of post-discharge venous thromboembolism in patients with rheumatoid arthritis undergoing knee or hip arthroplasty. Is prolonged thromboprophylaxis warranted or dangerous?
}

Venous thromboembolism (VTE) - that is, deep venous thrombosis (DVT) and pulmonary embolism (PE) - are common complications after major hip or knee surgery. Without thromboprophylaxis, the incidence of DVT in patients undergoing major orthopaedic surgery is more than $50 \%$, and fatal PE is reported to occur in $1-6 \%$ of these patients. ${ }^{1}$ These data are based on investigations in which predominantly osteoarthritis (OA) patients were studied. Only a few (small) studies were performed in rheumatoid arthritis (RA) patients: Abernethy reports an incidence of DVT of more than $70 \%$ and overall incidence of PE of approximately $2 \%$ in RA patients who have undergone a knee arthroplasty, ${ }^{2}$ and Kelly reports, in his review, ${ }^{3}$ an incidence of fatal PE of approximately $1 \%$ in RA patients undergoing total hip replacement and not receiving thromboprophylaxis (table 1 ). The risk of developing DVT seems to be similar for RA patients undergoing total hip replacement and those undergoing knee replacement surgery. ${ }^{4}$

Data are conflicting regarding the risk for developing VTE for RA patients in comparison with OA patients undergoing major orthopaedic surgery. Similar incidences of VTE have been reported for OA patients undergoing major orthopaedic surgery, ${ }^{56}$ whereas one large retrospective study indicated a possibly lower rate of thromboembolic complications in RA patients (table 1$).{ }^{7}$ In this investigation, inhospital morbidity and mortality of 721 RA patients and 8859 OA patients, who underwent an elective hip replacement operation, were analysed retrospectively. The incidence of thromboembolic events was $0.3 \%$ in the RA group versus $1.2 \%$ in the OA group $(p=0.07)$. At first sight, these very low incidences of VTE are remarkable, but they are probably related to a very short observation period and underdiagnosing, by only observing clinical VTE. Hence, the incidence of VTE seems to be underestimated in this study.

Altogether, it remains unknown whether there is a difference in VTE risk between RA and OA patients and obviously, more reliable data should come from (prospective) investigations with adequate end point assessment.

\section{Development of venous thrombosis during/after (orthopaedic) surgery}

Hypercoagulability, caused by tissue damage and intraoperative and postoperative stasis, is a common pathogenic factor for the development of DVT, in all surgical patients.

Additional pathogenic factors play an important part in major orthopaedic procedures. The femoral vein is distorted and kinked during total hip replacement. This leads to stasis of blood and to vessel wall damage, which promotes fibrin formation. In addition, it leads to a local exhaustion of tissue plasminogen activator (t-PA), the activator of the fibrinolytic system. ${ }^{8}$ This results in an insufficient suppression of the local fibrin formation that occurs during the operation.

During total knee arthroplasty, tourniquets are usually applied to reduce blood loss and this results in stasis of blood and anoxia in the distal portion of the leg, which also leads to an exhaustion of endothelial t-PA stores and inadequate inhibition of fibrin formation.

Patients who have undergone major orthopaedic surgery develop swelling in the operated region, partly because of inflammatory reactions and partly because of wound haematomas, which may result in leg vein compression and stasis of blood.

The exhaustion of t-PA stores, in addition to local venous endothelial damage and blood stasis, leads to an increased perioperative fibrin formation and may explain the high incidences of VTE in major orthopaedic surgery.

The peak incidence of DVT is observed around the fifth postoperative day. ${ }^{49}$ After the first postoperative week a second activation of coagulation occurs, as demonstrated by an increase of thrombin-antithrombin III complexes and D-dimer, markers of coagulation activation, which may persist up to six weeks or longer. ${ }^{10}$ The pathophysiological mechanism for this activation is not known, but it might be partly attributable to a (relative) immobilisation of the patient after hospital discharge in addition to the discontinuation of pharmacological thromboprophylaxis.

Need for thromboprophylaxis during hospitalisation Obviously, thromboprophylaxis is mandatory in patients undergoing major orthopaedic surgery and presently, during the hospitalisation period, low molecular weight heparin (LMWH) is the most commonly applied thromboprophylactic agent in the majority of the orthopaedic surgery units in Europe. The evidence for the efficacy and safety of LMWH is derived from (large) trials in which predominantly OA patients were studied. However, although no conclusions for RA patients could be drawn in view of the very limited number of RA patients studied, LMWH is also commonly used in RA patients as thromboprophylaxis.

Numerous investigations and several meta-analyses have demonstrated the efficacy and safety of LMWH in the prevention of postoperative thromboembolic events after major orthopaedic surgery (table 2).$^{11-13}$ Several studies have compared LMWH with unfractionated heparin in patients undergoing major orthopaedic surgery, indicating an overall risk reduction of approximately $25 \%$ for the

Table 1 Inhospital venous thromboembolism, without thromboprophylaxis, in major orthopaedic surgery patients

\begin{tabular}{|c|c|c|c|c|c|}
\hline Study & Patients (n) & Operation & Patients category & $\begin{array}{l}\text { Deep vein } \\
\text { thrombosis (\%) }\end{array}$ & $\begin{array}{l}\text { Pulmonary } \\
\text { embolism (\%) }\end{array}$ \\
\hline \multicolumn{6}{|c|}{ Venographically assessed DVT } \\
\hline Salzman and Hirsh ${ }^{\star 1}$ & - & THR & not reported & 50 & $1-6$ \\
\hline Kelly $^{\star 3}$ & - & THR & RA & 50 & 1 \\
\hline Abernethy ${ }^{\star 2}$ & - & TKR & $\mathrm{RA}$ & 70 & 2 \\
\hline Stringer ${ }^{5}$ & 55 & THR & not reported & 56 & 2 \\
\hline \multicolumn{6}{|l|}{ Clinically assessed DVT } \\
\hline White et al & 8859721 & THR THR & OA RA & $1.2 \dagger 0.3 \dagger$ & \\
\hline
\end{tabular}

DVT=deep venous thrombosis. THR=total hip replacement. RA=rheumatoid arthritis. TKR=total knee replacement. OA=osteoarthritis. ${ }^{\star}$ Review. $\dagger$ Clinically apparent deep venous thrombosis and pulmonary embolism. 
Table 2 Inhospital venous thromboembolism, with thromboprophylaxis, in major orthopaedic surgery patients

\begin{tabular}{|c|c|c|c|c|c|c|}
\hline Study & Patients (n) & Operation & Patients category & $\begin{array}{l}\text { Thrombo- } \\
\text { prophylaxis }\end{array}$ & $\begin{array}{l}\text { Deep vein } \\
\text { thrombosis (\%) }\end{array}$ & $\begin{array}{l}\text { Pulmonary } \\
\text { embolism (\%) }\end{array}$ \\
\hline \multicolumn{7}{|c|}{ Venographically assessed DVT } \\
\hline Sharrock et al $l^{6}$ & 436 & TKR & OA $(n=382) ; R A(n=54)$ & aspirin & 4239 & not reported \\
\hline Nurmohamed et al $l^{\star 11}$ & 672 & THR & not reported & LMWH & 14 & 2 \\
\hline Swierstra $e t a l^{14}$ & 101 & THR & OA $(n=87) ;$ RA $(n=14)$ & $\mathrm{OAC}$ & $23+$ & 0 \\
\hline Howard et $a l^{13}$ & 1599 & TKR & not reported & LMWH & 32 & 0.3 \\
\hline \multicolumn{7}{|l|}{ Clinically assessed DVT } \\
\hline Leclerc et $a l^{16}$ & 1141842 & THR TKR & not reported & LMWH LMWH & 1.31 .8 & 0.50 .4 \\
\hline
\end{tabular}

${ }^{\star}$ Meta-analysis. LMWH=low molecular weight heparin. OAC $=$ oral anticoagulants. $\nmid$ Overall incidence. DVT=denotes deep venous thrombosis. THR $=$ denotes total hip replacement. $\mathrm{RA}=$ denotes rheumatoid arthritis. $\mathrm{TKR}=$ denotes total knee replacement. $\mathrm{OA}=$ denotes osteoarthritis.

development of DVT and an approximately 50\% risk reduction for PE, when comparing $\mathrm{LMWH}$ with unfractionated heparin, without an increase of major bleeding complications. ${ }^{113}$

However, there is still a considerable rate of symptomless DVT, approximately $15-20 \%$ at the end of the hospital stay. ${ }^{12}$

\section{Is post-discharge thromboprophylaxis needed?}

Despite this high residual rate of approximately 15-20\% and laboratory evidence of continuing coagulation activation, there is no consensus whether or not prophylaxis should be continued after hospital discharge in OA or RA patients who have undergone major orthopaedic surgery.

Reduction of post-discharge VTE by anticoagulants should be carefully weighed against risk of bleeding and the concomitant costs. To date, no conclusive evidence, particularly for RA patients, is available and presently, many orthopaedic surgeons in the Netherlands continue thromboprophylaxis after hospital discharge for six weeks up to three months, for which oral anticoagulants, mostly acenocoumarol, are used. This practice is based on one small scale investigation in which a total of 101 patients, 87 OA patients and 14 RA patients, undergoing elective total hip replacement, were studied. All patients received oral anticoagulants as thromboprophylaxis during the hospital stay, which was stopped at the time of the hospital discharge. This study indicated a high rate of postdischarge non-fatal symptomatic PE, which was observed in three of 55 patients $(7 \%)$ with negative radionuclide venography 10 days after the operation. ${ }^{14}$ The major drawback of this investigation is that the applied detection method, radionuclide venography, is not an adequate screening test for detecting (symptomless) DVT, which can only be detected properly by contrast venography. ${ }^{15}$ Hence, the PE observed in this investigation might have come from patients with a false negative radionuclide venography and therefore, the true post-discharge incidence of PE might be overestimated.

Recently, a much lower incidence of post-discharge PE was reported. ${ }^{16}$ In this large open trial the occurrence of post-discharge clinically overt VTE in patients not receiving prolonged prophylaxis was investigated. A total of almost 2000 patients (predominantly OA), undergoing hip or knee arthroplasty, were studied and clinically overt VTE was observed in 40 patients (2\%). DVT was detected in 25 patients, non-fatal PE in 14 patients and one patient suffered from a fatal PE. To date, no data regarding the incidence of post-discharge VTE in RA patients have been published.

Thus far, the routine use as well as the duration of postdischarge anticoagulation with oral anticoagulants has never been validated adequately and consequently, postdischarge anticoagulation is not applied by all orthopaedic surgeons. ${ }^{17}$

Bleeding risks caused by prolonged (post-discharge) anticoagulation with oral anticoagulants have never been resolved properly for major orthopaedic surgery patients.
Investigations in other patient categories (for example, treatment of VTE) indicate, per treated patient, a major bleeding risk (requiring hospitalisation) of $1-8 \%$ and a fatal bleeding rate of $0.5-4.8 \% .{ }^{18-23}$ An important point of consideration, is the additional bleeding risk if a nonsteroidal anti-inflammatory drug (NSAID) is used in combination with oral anticoagulants. ${ }^{24}$ For instance, the relative risk for a gastrointestinal bleeding or perforation is more than six times increased when a NSAID is used in addition to oral anticoagulants. ${ }^{25}$ These bleeding risks also favour withholding prolonged thromboprophylaxis. Additional arguments are:

(1) the clinical significance of symptomless DVT is not known, although it is generally accepted, that some will lead to symptomatic DVT and PE, ${ }^{9627}$ and others to the post-thrombotic syndrome ${ }^{28}$

(2) immobilisation, an important risk factor for the development of VTE during hospital stay, ${ }^{29}$ has only a limited significance at the time of the hospital discharge, as most patients are then ambulant

(3) a thromboprophylactic effect has been suggested for NSAIDs, ${ }^{30}$ and therefore, prolonged prophylaxis would not be required for RA patients as most are using NSAIDs.

Altogether, it seems that the balance between benefit and risk for prolonged (post-discharge) anticoagulation, particularly for RA patients, has not yet been elucidated.

\section{Do aspirin and other NSAIDs have an effect on venous thromboembolism?}

Aspirin irreversibly inhibits cyclooxygenase by acetylation whereas other NSAIDs produce a reversible inhibition by competing, with the substrate arachidonic acid, for the active site on the enzyme. The major mechanism for the well established (arterial) anti-thrombotic effect of aspirin is mediated through its ability to irreversibly block the synthesis of platelet thromboxane $\mathrm{A}_{2}{ }^{31}$

Classically the arterial thrombus is largely composed of platelets and fibrin and therefore, arterial thrombi are known as white thrombi. Venous thrombi have a much richer content of erythrocytes and are therefore known as red thrombi. Hence, it is conceivable that, from a pathophysiological point of view, aspirin (and other NSAIDs), because of their inhibition of platelet aggregation, have a more pronounced (inhibitory) effect on the development of arterial thrombi compared with venous thrombi.

For NSAIDs also a prophylactic effect on the development of VTE has been hypothesised.

EXPERIMENTAL ANIMAL INVESTIGATIONS

One experimental animal study indicated a moderate, inhibitory, effect of NSAIDs on VTE. ${ }^{30}$ In this investigation, Doutremepuich and coworkers investigated the effect of NSAIDs on laser induced venous and arterial thrombosis in a rat model. For several NSAIDs, a significantly higher number of laser beams was required to induce arterial thrombosis, compared with control ( $\mathrm{NaCl} 0.9 \%)$, 
whereas this effect was not observed for the induction of venous thrombi. The number of emboli generated from these thrombi as well as the duration of embolisation were, for both arterial and venous thrombi, significantly decreased by NSAIDs compared with control. This experiment suggests that NSAIDs have a more pronounced inhibitory effect on the development of arterial thrombi than on venous thrombus formation and a similar effect on arterial and venous emboli.

\section{CLINICAL INVESTIGATIONS}

A meta-analysis of earlier venous thromboprophylaxis trials among surgical and medical patients, in which antiplatelet agents (primarily aspirin) were investigated as thromboprophylactic agents, indicated some beneficial effect of aspirin against DVT in elective orthopaedic surgery. ${ }^{32}$ Thirteen elective orthopaedic surgery trials were considered for this analysis and encompassed a total of 863 patients. Antiplatelet therapy was administered to 427 patients, whereas there were 436 control patients. DVT was detected in 232 control patients $(53.2 \%)$ and in 160 antiplatelet treated patients (37.5\%, 49\% odds reduction). Although the reduction seems substantial, these results must be interpreted cautiously as most studies that were included in this meta-analysis, suffered from methodological deficiencies, such as an open study design and inadequate end points. Hence, this meta-analysis cannot be considered conclusive regarding the effect of aspirin on the prevention of VTE. ${ }^{33}$ A thromboprophylactic effect on VTE of NSAIDs, other than aspirin, has never been proved in recent clinical investigations. This is probably related to the fact that in most thromboprophylaxis trials NSAIDs were either stopped, or their use was strongly discouraged or patients using NSAIDs were excluded. Altogether, there seems no clinically relevant effect of aspirin (and other NSAIDs) in the prevention of venous thromboembolism, in contrast with the well established efficacy of aspirin in preventing arterial thrombosis. ${ }^{27}$

NSAIDs are used by most RA patients and, to a lesser extent, by OA patients. They are stopped postoperatively in the majority of OA patients whereas they are continued, and still used after hospital discharge, in RA patients. Therefore, the balance of benefit-that is, reduction of post-discharge thromboembolism-and risk-that is, induction of bleeding complications-could be different for RA patients compared with OA patients.

\section{Post-discharge thromboprophylaxis}

Recently, four randomised double blind studies investigating extended prophylaxis with LMWH after hospital discharge were reported..$^{35-38}$ The investigations had a comparable design involving post-discharge randomisation to either additional LMWH for a period of up to five weeks, or placebo. Almost 900 OA patients undergoing total hip replacement were investigated and large risk reductions of $40-60 \%$, with residual DVT incidences of 4 up to $19 \%$, during the post-hospitalisation period, were observed

Table 3 Post-discharge venous thromboembolism in patients undergoing major hip replacement

\begin{tabular}{|c|c|c|c|c|c|c|}
\hline \multirow[b]{2}{*}{ Study } & \multirow{2}{*}{$\begin{array}{l}\text { Patients } \\
(n)^{\star}\end{array}$} & \multicolumn{2}{|c|}{$\begin{array}{l}\text { Deep vein } \\
\text { thrombosis (\%) }\end{array}$} & \multirow{2}{*}{$\begin{array}{l}\text { Risk } \\
\text { reduction } \\
(\%)\end{array}$} & \multicolumn{2}{|c|}{$\begin{array}{l}\text { Pulmonary } \\
\text { embolism (\%) }+\end{array}$} \\
\hline & & Placebo & $L M W H$ & & Placebo & $L M W H$ \\
\hline Dahl et $a l^{35}$ & 227 & 32 & 19 & 39 & $3 \ddagger$ & 0 \\
\hline Lassen et $a b^{36}$ & 215 & 12 & 4 & 63 & 0 & 0 \\
\hline Planes et a $b^{37}$ & 173 & 19 & 7 & 63 & 0 & 0 \\
\hline Bergqvist et $a l^{88}$ & 233 & 37 & 18 & 52 & 2 & 0 \\
\hline
\end{tabular}

${ }^{\star}$ With evaluable venograms. †Confirmed by either lung scan, angiography or necropsy. $\ddagger$ Including one fatal pulmonary embolism. (table 3). There was no increased risk of bleeding complications or other adverse events in the LMWH groups. However, only a very limited number of RA patients were studied (for example, only 1 of 179 patients studied in the trial of Planes and coworkers, ${ }^{37}$ had RA) and therefore, these encouraging results cannot be extrapolated to RA patients.

In addition, a comparison with oral anticoagulants, the commonly applied drug for prolonged thromboprophylaxis in the Netherlands, is lacking and finally, the optimal duration of post-discharge thromboprophylaxis is not known.

Therefore, extended pharmacological prophylaxis of VTE in RA patients, undergoing major orthopaedic interventions, continues to be a serious matter of debate, which only can be solved by an appropriate trial.

\section{Conclusions}

VTE is an important postoperative complication of major orthopaedic surgery in OA and RA patients, albeit that, in RA patients, the risk for developing VTE might be lower than in OA patients. LMWH effectively reduces the incidence of VTE during hospital stay in OA patients. LMWH is also commonly used as postoperative thromboprophylaxis in RA patients but this has never been validated.

The incidence of post-discharge VTE in OA patients seems to be substantial, and there is accumulating evidence that this can be safely lowered by prolonged administration of LMWH.

No data are available regarding the magnitude of post-discharge VTE in RA patients. The risk of RA patients might be different in comparison with OA patients in view of (a) an a priori possibly lower incidence of VTE during hospital stay and (b) post-discharge use of NSAIDs, which is accompanied by an increased bleeding risk if they are used in combination with anticoagulants. Hence, the balance of benefit and risk might be quite different for RA patients in comparison with OA patients.

Clearly there is a need for a randomised placebo controlled trial with LMWH and objective end point assessment (that is, venography) that investigates this topic. Only after the results of such an investigation become available, can definitive recommendations regarding extended thromboprophylaxis for RA patients undergoing major orthopaedic surgery be given - that is, whether it is warranted or dangerous.

M T NURMOHAMED Department of Rheumatology, Slotervaart Hospital and fan van Breemen Institute, Amsterdam, the Netherlands

W F LEMS

B A C DIJKMANS

Department of Rheumatology, Slotervaart Hospital, Department of Rheumatology, Free University Hospital, and fan van Breemen Institute, Amsterdam, the Netherlands

1 Salzman EW, Hirsh J. The epidemiology, pathogenesis and natural history of venous thrombosis. In: Colman RW, Hirsh J, Marder VJ, Salzman EW, eds. Hemostasis and thrombosis: basic principles and clinical practice. Philadelphia: Hemostasis and thrombosis: basic pr

2 Abernethy PJ. Surgery of the rheumatoid knee. Ann Rheum Dis 1990;49:830-6.

3 Kelly IG. Surgical treatment of the rheumatoid hip. Ann Rheum Dis 1990; 49:858-62.

4 Cooke TDV. Orthopaedic conditions and surgery. Curr Opin Rheumatol 1992;4:179-83.

5 Stringer MD, Steadman CA, Hedges AR, Thomas EM, Morley RM, Kakkar VV. Deep vein thrombosis after elective knee surgery - an incidence study in 312 patients. J Bone Joint Surg Br 1989;71:492-7.

6 Sharrock NE, Hargett MJ, Urquhart B, Peterson MGE, Ranawat C, Insall J, et al. Factors affecting deep vein thrombosis rate following total knee arthroplasty under epidural anesthesia. J Arthroplasty 1993;8:133-9.

7 White RH, McCurdy SA, Marder RA. Early morbidity after hip replacement: rheumatoid arthritis versus osteoarthritis. J Gen Intern Med 1990;5:304-9.

8 Bachmann F, Leyvraz PF. Low-molecular-weight heparins for prevention of venous thromboembolism following orthopedic surgery. In: Bounameaux $\mathrm{H}$, ed. Low molecular weight heparins in prophylaxis and therapy of thromboembolic diseases. New York: Marcel Dekker Inc, 1994:187-212. 
9 Kakkar VV. Low molecular weight heparins: prophylaxis of venous thromboembolism in surgical patients. Semin Hematol 1997;4 (suppl 4):9-19.

10 Dahl OE, Aspelin T, Amersen H, Seljeflot I, Kierulf P, Ruyter R, et al. Increased activation of coagulation and formation of late deep venous thrombosis following discontinuation of thromboprophylaxis after hip replacement surgery. Thromb Res 1995;4:299-306.

11 Nurmohamed MT, Rosendaal FR, Büller HR, Dekker E, Hommes DW, Vandenbroucke JP, et al. Low molecular weight heparin in general and orthopedic surgery: a meta-analysis. Lancet 1992;340:152-6.

12 Nurmohamed MT, Cate H ten, Cate JW ten. Low molecular weight heparin(oid)s. Drugs 1997;53:736-51.

13 Howard AW, Shawn SD. Low molecular weight heparin decreases proximal and distal deep venous thrombosis following total knee arthroplasty. and distal deep venous thrombosis

14 Swierstra BA, Stibbe J, Schouten HJA. Prevention of thrombosis after hip arthroplasty: a prospective study of preoperative oral anticoagulants. Acta

Orthop Scand 1988;59:139-43.
15 Büller HR, Lensing AWA, Hirsh J, ten Cate JW. Deep vein thrombosis: new non-invasive diagnostic tests. Thromb Haemost 1991;66:133-7.

16 Leclerc JR, Gent M, Hirsh J, Geerts WH, Ginsberg J. The incidence of symptomatic venous thromboembolism during and after prophylaxis with enoxaparin: a multi-institutional cohort study in patients who underwent hip or knee arthroplasty. Arch Intern Med 1998;158:873-8.

17 ten Cate JW, Prins MH. Major orthopaedic surgery and post-discharge DVT. Lancet 1996;348:209-10.

18 Hull R, Hirsh J, Jay R, Carter C, England C, Gent M, et al. Different intensities of oral anticoagulant therapy in the treatment of proximal vein thrombosis. N Engl J Med 1982;307:1676-81.

19 Landefeld CS, Goldman L. Bleeding in outpatients treated with warfarin: incidence and prediction by factors known at the start of outpatient therapy. Am J Med 1989;87:144-52.

20 Landefeld S, Beyth RJ. Anticoagulant-related bleeding: clinical epidemiology, prediction and prevention. Am J Med 1993;95:315-28.

21 Van der Meer FJM, Rosendaal FR, Vandenbroucke JP, Briët E. Bleeding complications in oral anticoagulant therapy. Arch Intern Med 1993;153: 1557-62.

22 Palareti G, Leali N, Coccheri S, Poggi M, Manotti C, D’Angelo A, et al. Bleeding complications of oral anticoagulant treatment: an inceptioncohort, prospective collaboration study (ISCOAT); Lancet 1996;348:42338.

23 Kuijer PMM, Hutten BA, Prins MH, Büller HR. Prediction of the risk of bleeding during anticoagulant treatment for venous thromboembolism. In: Kuijer PMM. Diagnostic and therapeutic management of pulmonary embolism. University of Amsterdam: Academic Thesis, 1997:131-41.

24 Schulman S. Interaction of ibuprofen and warfarin on primary hemostasis. Br J Rheumatol 1989;28:46-9.
25 Rodriguez L, Jick H. Risk of upper gastrointestinal bleeding and perforation associated with individual non-steroidal anti-inflammatory drugs. Lancet 1994;343:769-72.

26 Bergqvist $\mathrm{D}$. The postdischarge risk of venous thromboembolism after hip replacement: The role of prolonged prophylaxis. Drugs 1996;52 (suppl 7):55-9.

27 Hirsh J. Evidence for the needs of out-of-hospital thrombosis prophylaxis: introduction. Chest 1998;114 (suppl):113-4S

28 Davidson BL. Out-of-hospital prophylaxis with low-molecular-weight heparin in hip surgery. Chest 1998;114 (suppl):130-2S

29 Thrombosis Risk Factors (THRIFT) Consensus Group. Risk of and prophylaxis for venous thromboembolism in hospital patients. BMJ 1992;305:567-74.

30 Doutremepuich F, Aguejouf O, Imbault P, Azougagh Oualane F, Doutremepuich C. Effect of low molecular weight heparin/non-steroidal antiinflammatory drugs association on an experimental thrombosis induced by laser. matory drugs association on an
Thromb Res 1995;77:311-19.

31 Hirsh J, Dalen JE, Fuster V, Harker LB, Patrono C, Roth GJ. Aspirin and other platelet-active drugs: The relationship between dose, effectiveness and side effects. Chest 1995;108 (suppl):247-57.

32 Antiplatelet Trialists' Collaboration. Collaborative overview of randomised trials of antiplatelet therapy. III. Reduction in venous thrombosis and pulmonary embolism by antiplatelet prophylaxis among surgical and medical patients. BMJ 1994;308:235-46.

33 Cohen AT, Skinner JA, Kakkar VV. Antiplatelet treatment for thromboprophylaxis: a step forward or backwards. BMJ 1994;309:121317.

34 Antiplatelet Trialists, Collaboration. Collaborative overview of randomised trials of antiplatelet therapy. I. Prevention of death, myocardial infarction, and stroke by prolonged antiplatelet therapy in various categories of and stroke by prolonged antip

35 Dahl OE, Andreassen G, Aspelin T, Müller C, Mathiesen P, Nyhus S, et al. Prolonged thromboprophylaxis following hip replacement surgery. Results of a double-blind, prospective, randomized placebo-controlled study with Dalteparin $\left(\right.$ Fragmin $\left.^{\mathrm{R}}\right)$. Thromb Haemost 1997;77:26-31

36 Lassen MR, Borris LC, Anderson BS, Jensen HP, Bro HPS, Andersen G, et al. Efficacy and safety of prolonged thromboprophylaxis with a low molecular weight heparin (Dalteparin) after total hip arthroplasty. Thromb Res 1998;89:281-7.

37 Planes A, Vochelle N, Darmon JY, Fagola M, Bellaud M, Huet Y. Risk of deep-venous thrombosis after hospital discharge in patients having undergone total hip replacement: double blind randomised comparison of enoxaparin versus placebo. Lancet 1996;348:224-8.

38 Bergqvist D, Benoni G, Björgell O, Fredin $\mathrm{H}$, Hedlundh U, Nicolas S, et al. Low-molecular-weight heparin (enoxaparin) as prophylaxis against venous thromboembolism after total hip replacement. N Engl J Med 1996;335: 696-700. 